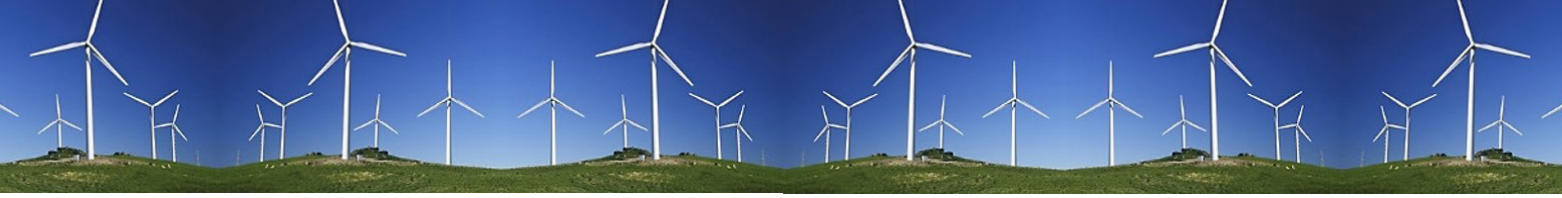

\begin{abstract}
Artículo de investigación E16A13. * Proyecto "Nuevas formas de aplicación PSM para analizar el impacto de las ayudas públicas a la I+D empresarial en Méjico: un estudio macroeconómico". Instituto de Investigaciones Económicas de la Universidad Nacional Autónoma de México. Recibido: 15.11.2019. * Aprobado versión final: 26.12.2019. * JEL: D2, J21, O31, O33. Pp. 55-89. * doi: 10.33571/teuken.v11n16a3
\end{abstract}

\title{
Innovación y empleo. Paradojas sociales y económicas.
}

\section{Innovation and employment.}

\author{
Social and economic paradoxes.
}

\section{Joost Heijs - Guillermo Arenas Díaz ESPAÑA}

\begin{abstract}
Resumen: La relación entre la innovación y el empleo es un tema debatido desde los inicios del capitalismo. A menudo, domina la visión pesimista, donde la nueva tecnología tiende a desplazar la mano de obra existente, como es el caso de la actual discusión sobre la robotización y la inteligencia artificial. Sin embargo, la evidencia empírica muestra que las tasas de empleo siguen creciendo a pesar de la introducción de nuevas máquinas. El objetivo del artículo es revisar las tendencias y teorías respecto a la relación innovación versus empleo tanto en términos cuantitativos como cualitativos. Además, se discute de forma crítica el impacto que tienen las nuevas tecnologías sobre las relaciones laborales en términos del bienestar social y la distribución internacional del empleo y la riqueza.
\end{abstract}

Palabras claves: innovación; empleo; cambio tecnológico sesgador de habilidades y rutinas; polarización de empleo.

Abstract: The relationship between innovation and employment is a topic debated since the beginning of capitalism. The pessimistic view often dominates where new technology tends to displace labor, as it is the case in the current discussion on robotization and artificial intelligence. However, empirical evidence shows that employment rates continue growing despite the introduction of new machines. The goal of the article is to review trends and theories regarding the relationship between innovation versus employment, in both quantitative and qualitative terms. In addition, the impact of new technologies on labor relations in terms of social welfare and the international distribution of employment and wealth are critically discussed.

Keywords: innovation; employment; skill and routine based technical change; labour polarization.

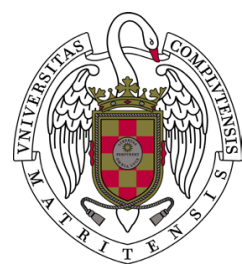

Joost Heijs es Doctor en Ciencias Económicas y Empresariales de la Universidad Complutense de Madrid, Co-director de la Asociación Latino-lbero Americana de Gestión de Tecnología, Director del Instituto de Análisis Industrial y Financiero y Profesor titular e investigador del Departamento de Economía Aplicada, Estructura e Historia en la Facultad de Ciencias Económicas y Empresariales de la Universidad Complutense de Madrid.

Contacto: joost@ccee.ecm.es

Guillermo Arenas Díaz es Licenciado y Magíster en Economía de la Universidad Nacional Autónoma de México. Actualmente, adelanta estudios de doctorado en Ciencias Económicas en la Universidad Complutense de Madrid.

Contacto: memoare20@gmail.com

Teuken Bidikay Vol. 11 № 16 (Medellín, Colombia) * Ene-Jun 2020. ISSN: 2215-8405 - e-ISSN 2619-1822. Pp. 55-89 


\section{Inovação e emprego.}

Paradoxos sociais e econômicos.

Resumo: A relação entre inovação e emprego tem sido um tópico de debate desde o início do capitalismo. A visão pessimista costuma dominar, onde as novas tecnologias tendem a deslocar o trabalho existente, como é o caso da atual discussão sobre robótica e inteligência artificial. No entanto, evidências empíricas mostram que as taxas de emprego continuam a crescer, apesar da introdução de novas máquinas. $\mathrm{O}$ objetivo do artigo a seguir é revisar as tendências e teorias relacionadas à inovação em relação ao emprego em termos quantitativos e qualitativos. Além disso, o impacto das novas tecnologias nas relações de trabalho em termos de bem-estar social e distribuição internacional de emprego e riqueza.

Palavras-chave: inovação; emprego; mudança tecnológica de pular habilidades e rotinas; polarização de emprego.

\section{Introducción}

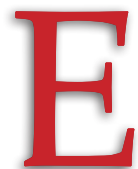

I impacto del cambio tecnológico en el mercado laboral genera controversia social y resurge cada vez que una nueva revolución de la tecnología aumenta de forma drástica la productividad con un alto potencial de reducir el empleo. Las posibles implicaciones de las nuevas formas de "robotización", originadas por los avances en la inteligencia artificial, en el futuro, en términos de empleo, han generado un fuerte debate social porque tal combinación permite una intensificación sustancial del proceso de automatización apuntando a una reducción importante en la demanda de empleo (Dorn, 2016; Arntz, et al., 2017; Acemoglu \& Restrepo, et al. 2017). De hecho, Mc Kinsey (2017)' indica que la automatización podría destruir, aproximadamente, entre el $40 \%$ y $50 \%$ del empleo.

En realidad, el origen de este debate social se remonta al inicio de la industrialización, cuando los machine-breakers, es decir, los trabajadores altamente calificados del sector textil perdieron su empleo y se rebelaron contra los empresarios. ${ }^{2}$ Sin embargo, tales movimientos solo lograron una

1 Una estimación basada en el análisis de 54 países, incluido Méjico, donde el 52\% del empleo puede ser automatizado.

2 La innovación de proceso fue la actividad más notable durante la primera revolución industrial con un enorme impacto en el empleo. Por ejemplo, las primeras máquinas del sector textil redujeron la demanda del empleo para producir 100 libras de algodón desde unas 50.000 (producción manual) a 2.000 horas (Maquina Crompton de 1780). Es decir, 24 de cada 25 trabajadores perdieron su trabajo, siendo además, en su mayoría trabajadores muy calificados, cuyos conocimientos y habilidades quedaron obsoletos. La maquinaria moderna de 1990 requiere solo 40 horas para hacer esta misma tarea. Es decir, desde el inicio de la industrialización la productividad del sector de textil se ha multiplicado por 1250 veces (Jenkins, 1994). En el sector de la agricultura se observa un aumento todavía mayor de la productividad durante el siglo XX en unas 3.500 veces según datos de la UNESCO (2005). 
desaceleración de la difusión de la innovación y no pudieron detener el progreso tecnológico en el largo plazo (Hobsbawm, 1952: 58). Resulta que el progreso tecnológico, ahorrador del empleo, es difícil de impedir porque si una empresa (país) renuncia a tales posibilidades de ganar eficiencia otra lo hará. Sin embargo, las ganancias en la eficiencia del sistema productivo han permitido reducir el horario laboral.

En el gráfico 1 se observa el alto número de horas laborales (en promedio, 2500 y 3000 horas en el año 1900) que necesitaban los trabajadores de los países más avanzados para mantener un nivel de vida digno (prevalecieron en los albores de la Revolución Industrial y durante la primera parte del siglo XX). Las horas laborales disminuyeron gradualmente a medida que los países alcanzaron niveles más altos de desarrollo (hasta unas 2000 horas al final de este siglo) (Maddison, 2001). En países avanzados la semana laboral se ha reducido hasta 38 horas en 2019 con respecto a la semana laboral de 64 horas en el año 1870; mientras que en los países menos desarrollados la semana laboral de 48 horas sigue siendo la más común (ILO, 2018). Dentro de los países de la OCDE, Méjico es el país con la semana laboral más intensiva (unas 44 horas semanales y 2.228 horas anuales), siendo una carga laboral parecida a la media simple de 1960 de los países incluidos.

Gráfico 1. Jornada semanal de países seleccionados (en horas)

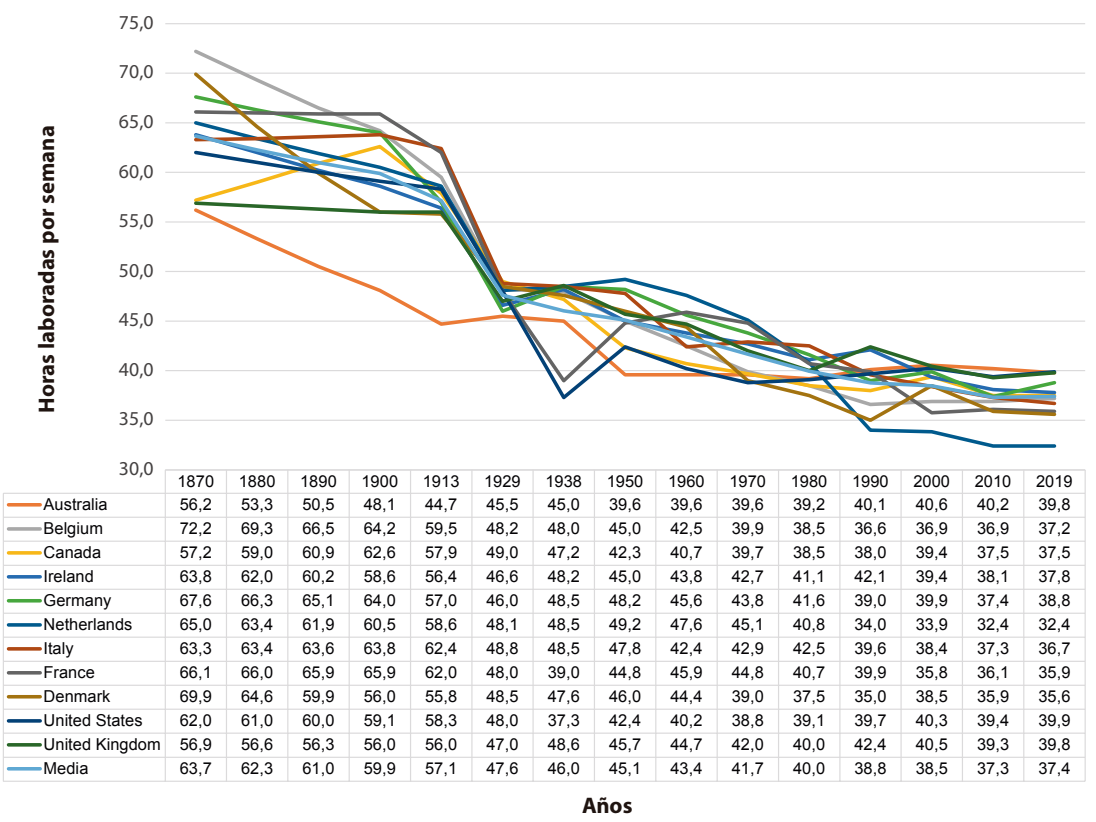

Fuente: Elaboración propia con datos de la OCDE (2019). 
Aunque en las últimas décadas, y especialmente durante la gran crisis, la reducción en el número de las horas laborales cesó, incluso se revirtió en algunos países, parece que la nueva revolución tecnológica basada en la robotización, en combinación con la Inteligencia Artificial (IA) y las impresoras 3D, apunta de nuevo a reducciones importantes en la demanda laboral. La idea de una semana laboral muy reducida ya lo había previsto John Maynard Keynes (1930), quien afirmó que la generación de sus nietos tendría una jornada laboral de solo 15 horas semanales. En aquella época tal predicción parecía extravagante y audaz, pero viéndolo en retrospectiva no parece tan desacertado.

Respecto a la relación entre la innovación y el empleo, o mejor dicho el desempleo, existen varias paradojas. La primera se refiere a la relación entre la innovación, la productividad y la demanda de empleo en términos cuantitativos. Aunque vivimos en una era en la que el progreso tecnológico avanza cada vez más rápido y las aplicaciones que incorpora la IA igualan o superan en algunas actividades el desempeño humano, también se observa una disminución del ritmo del crecimiento de la productividad (Brynjolfsson et al., 2017). Posiblemente, el efecto de las innovaciones de los sectores de Telecomunicaciones e Informática (TIC) ya no sea significativo sobre la productividad agregada, mientras que la IA, en combinación con la robotización, todavía no ha desarrollado todo su potencial.

Muchos autores (David, 1991; Pérez, 2002; Brynjolfsson et al., 2017) sostienen que el tiempo de difusión e integración de las nuevas tecnologías claves de producción, siendo las que aumentarían la productividad de cualquier sector, es muy largo y puede durar dos o tres décadas. De hecho, la difusión de las nuevas tecnologías claves tiene una forma de "S" donde inicialmente se observa una lenta difusión que, a partir de un cierto momento, se acelera y al final el aumento de su ciclo de vida, junto con la introducción de las nuevas aplicaciones, se ralentizan (Mansfield, 1963). El efecto de las nuevas revoluciones tecnológicas, u oleadas de innovaciones, sobre la productividad tienen una forma parecida, porque al final del ciclo de vida solo se introducen aplicaciones de menor valor añadido o importancia para el sistema productivo (Pérez, 2002). Es decir, el potencial de las tecnologías sobre la productividad refleja rendimientos marginales decrecientes.

Aparentemente, en este momento el sistema productivo está en la última fase del ciclo tecnológico anterior (las TIC) y todavía se encuentra en la fase de introducción de las nuevas tecnologías claves. Donde la IA, las impresoras 3D, el grafeno y otras nuevas posibles tecnologías claves todavía deben mostrar su utilidad, pero, sobre todo, difundir sus aplicaciones para un gran número de sectores, por lo que es pronto 
para valorar su impacto en el empleo. Una segunda paradoja es que las nuevas tecnologías y las ganancias en la productividad deberían mejorar la calidad de vida de los seres humanos y, por lo tanto, también la calidad del empleo, pero en realidad, las nuevas tecnologías generan dos efectos simultáneos sobre los trabajadores. Por un lado, un efecto muy negativo sobre la demanda de empleo con la expulsión de trabajadores calificados y no calificados.

Las nuevas tecnologías dejan obsoletas las habilidades de los trabajadores calificados, conllevando a su expulsión y pérdida de empleo, ya que no disponen de los nuevos conocimientos requeridos en los sectores emergentes, creando el llamado desempleo tecnológico ${ }^{3}$ (Schumpeter, 1942; Freeman \& Soete, 1987). Según Messenger (2018), ${ }^{4}$ Keynes consideraba esta situación de desempleo tecnológico solo como un problema a corto plazo de escasez (de la oferta laboral), donde la adaptación humana a esta situación es difícil pero no imposible. Aunque otros autores lo consideraban un cambio paradigmático difícil de superar por muchos de los trabajadores (Schumpeter,1942; Freeman \& Soete, 1987), especialmente, los de mayor edad. Es decir, una parte de los trabajadores calificados se convierte en no calificados con la pérdida correspondiente de su estatus social e ingresos económicos.

Así mismo, resalta el efecto de la polarización del mercado laboral, donde varios estudios destacan que el progreso tecnológico actual afecta especialmente a las personas de calificación intermedia (Ford, 2015). Una parte importante de los trabajos que exigen estudios de nivel intermedio se han visto desplazados por trabajos de baja calidad (Dorn, 2016). Según Ford (2015), el progreso tecnológico basado en la IA nos está acercando a un punto de inflexión crítico, previendo una estructura productiva significativamente menos intensiva en mano de obra, donde no solamente se ven amenazados los trabajos de rutinas simples sino también las tareas muy complejas basadas en decisiones predecibles a partir de una gran cantidad de información compleja y enrevesada, pero perfectamente codificable. Es decir, volvemos a la situación de los machine-breakers del siglo XVIII cuyo alto nivel de cualificación no evitaba una mecanización de sus tareas.

\footnotetext{
3 Como fue el caso de los trabajadores del sector textil a que se hace referencia en la primera nota a pie, los trabajadores cualificados del sector automóvil que perdieron su empleo debido a la introducción del fordismo; o los fotógrafos profesionales de los periódicos en Hong Kong cuyo trabajo ha sido como los repartidores de pizzas, ya que en el mundo de la prensa actual por internet más importante es tener una foto de forma rápido que uno de alta calidad "artística".
}

4 Presentado como un informe de la Oficina Internacional del Trabajo (OIT). 
Otra paradoja está relacionada con el sistema de producción capitalista moderno basado en la obsolescencia programada. Para mantener una demanda, y beneficios, a largo plazo las empresas diseñan productos con ciclos de vida cortos para que el consumidor siga comprando el mismo producto una y otra vez. De esta forma, se asegura la obtención continua de los beneficios y como consecuencia se mantiene un horario laboral excesivamente largo para producir una y otra vez lo mismo, en combinación con efectos negativos sobre el medioambiente y los recursos naturales. En este trabajo se debate el posible impacto de la innovación sobre la desigualdad y cómo se distribuye a nivel internacional el empleo y los beneficios en términos de eficiencia y productividad. Es decir, cuáles países se quedan con la riqueza creada por el progreso tecnológico.

Las implicaciones de estas dos paradojas, expulsión del empleo y la polarización se debaten en las secciones dos y tres de este trabajo; en las secciones cuatro, cinco y seis se presentan algunas paradojas de carácter social económico, revisando las contradicciones observadas en la relación con respecto a la innovación, la pobreza, la igualdad en un país y la convergencia económica entre países (desde el enfoque del mercado laboral). En la sección cuatro se exponen las oportunidades potenciales del progreso tecnológico respecto al bienestar social y reconciliación familiar.

\section{Innovación y la demanda cuantitativa de empleo: una visión teórica}

\section{Pensamiento neoclásico y la teoría evolucionista}

Existen diferentes escuelas de pensamiento económico que tratan de dar respuesta al efecto de la innovación en el empleo en términos cuantitativos, tales como los neoclásicos, la teoría keynesiana y las ideas evolucionistas, representadas por Schumpeter y Christopher Freeman. El pensamiento clásico introduce el concepto laissez-faire, suponiendo que la mejor forma de asignar los recursos, incluso el empleo, sería a través de un mercado libre sin la intervención del Estado. Según esta corriente se genera, gracias a la mecanización de la producción, una reducción de los precios, lo que da como resultado un mayor bienestar general a largo plazo. Sin embargo, David Ricardo (1821), uno de los máximos representantes del pensamiento económico clásico, pone en duda el efecto positivo de la innovación en el empleo. De hecho, este autor cuestiona fuertemente los beneficios de largo plazo que podría tener la innovación tecnológica y enfatiza en la disminución del empleo que 
se produce tras la introducción de las nuevas tecnologías. Por otro lado, Marx asegura que el avance tecnológico es intencionalmente impuesto por los capitalistas para aumentar el desempleo como medio de control de la mano de obra y para mantener los costos salariales a un nivel bajo.

El pensamiento neoliberal se puede considerar como una escuela predominante en el debate sobre la relación entre innovación y empleo. De hecho, los mecanismos de autoajuste, basados en la Ley de Say, donde cada oferta crea su propia demanda, se impregnaron en la disciplina económica. Según esta corriente, el mecanismo de equilibro se basa en la posibilidad de sustitución perfecta entre capital y trabajo. Se supone que el desempleo generado por la innovación sería una situación temporal del desequilibrio, donde la oferta de empleo generará una nueva demanda debido a un ajuste de los salarios a la baja. Una de las principales escuelas de pensamiento económico que pone en duda los mecanismos de autorregulación neoclásicos es el pensamiento keynesiano, criticando fuertemente uno de los principales pilares de la escuela neoliberal, la Ley de Say. Según Keynes, esta Ley solo funciona si existe una situación de pleno empleo, la cual raras veces llega a ocurrir. Keynes sostiene que el desempleo no es un problema de oferta, sino de demanda, por lo que no puede ser resuelto mediante un ajuste salarial en el mercado de trabajo. Los seguidores de la escuela keynesiana no ponen mucha atención en el cambio tecnológico como generador del desempleo. El mecanismo de demanda detrás de dicha escuela asume que en tiempos de crisis las expectativas de ganancias futuras son más bajas, lo que lleva a menores inversiones por parte de los empresarios que hace que la demanda se contraiga, esto a su vez desestimula la producción y, por lo tanto, el desempleo aumenta. En tiempos de recuperación, dicho mecanismo se invierte, las expectativas de ganancias mejoran, por lo que se genera un mayor nivel de inversión de los empresarios que estimula una mayor producción y, en consecuencia, un mayor empleo. Lo que no queda claro de esta teoría son las expectativas que generan la inversión. Keynes se lo atribuye a los "espíritus animales" un concepto derivado de la psicología social. ${ }^{6}$

5 Keynes considera los "espíritus animales" como las emociones que afectan el comportamiento económico y social del ser humano que no depende de la racionalidad. Su comportamiento depende más del optimismo espontáneo que de una expectativa matemática, ya sea moral, hedonista o económica. Tal comportamiento irracional no ponderado de los beneficios cuantitativos esperados multiplicados por las probabilidades cuantitativas sería el mencionado "'espíritus animales" (Keynes, 1936: 161-162).

6 Para más información de la teoría de Keynes, ver la Teoría General (1936)

Teuken Bidikay Vol. 11 № 16 (Medellín, Colombia) * Ene-Jun 2020. ISSN: 2215-8405 - e-ISSN 2619-1822. Pp. 55-89 
En contraste con la teoría keynesiana, Schumpeter asume que la innovación es la pieza clave detrás de las dinámicas y ciclos económicos. "Las revoluciones tecnológicas transforman las estructuras existentes de la industria al introducir nuevos métodos de producción (...) éstas crean nuevos productos, nuevas formas de organización, nuevas fuentes de oferta, nuevas rutas y mercados, entre otras cosas" (Schumpeter, 1942: 83). Detrás de esto, está el famoso concepto de Schumpeter de creación-destructiva, donde la innovación destruye las viejas formas de producción, mientras que las nuevas formas se van gestando. Por lo tanto, el sistema capitalista, visto bajo esta perspectiva, más allá de ser estático es dinámico. El desempleo bajo la perspectiva schumpeteriana se entiende como un resultado temporal inevitable, debido al cambio tecnológico. Se denomina como desempleo tecnológico causado por la discrepancia entre los conocimientos y habilidades de trabajadores expulsados de sectores tradicionales y las necesidades del capital humano que se requieren en los sectores innovadores emergentes. Como indica Rosenberg (1976), estas nuevas habilidades se pueden obtener a través de procesos de aprendizaje de largo plazo, debido al carácter acumulativo de las capacidades necesarias para manejar las nuevas tecnologías por parte de la fuerza laboral.

\section{Los efectos cuantitativos de la innovación sobre el empleo: los mecanismos de compensación}

Como se ha visto en la sección anterior, el efecto de la innovación sobre el empleo en términos cuantitativos es un tema que genera mucha controversia para los responsables de política pública y académicos en general, ya que la relación entre estas dos variables es difusa y poco entendida. De hecho, como veremos en esta sección la introducción de nuevas tecnologías implica un conjunto de consecuencias opuestas, de las cuales algunas implican efectos negativos sobre el empleo, mientras que otros implican la creación de nuevos puestos de trabajo. Aunque, el efecto neto al final del proceso no queda claro. Por eso, desde los inicios de la economía moderna como ciencia, los académicos han tratado de dar respuesta sobre los efectos negativos que tiene el cambio tecnológico en el empleo: innovación de proceso.

Esasíqueloseconomistasen el sigloXIXdesarrollaron un marco conceptual, que más tarde Marx denominó los "Mecanismos de Compensación" (MDC) que explican los procesos que mitigan el efecto negativo inicial del cambio tecnológico en el empleo. La teoría neoclásica ofrece una visión muy optimista respecto al impacto de la innovación basándose en los 
típicos supuestos poco realistas, como los mercados de libre competencia y la Ley de Say. Los neoclásicos reconocen dos efectos contradictorios en el empleo: el efecto de desplazamiento y de compensación. El primero de ellos se refiere a la reducción de las necesidades de input (empleo) por cada unidad de output (aumentando la productividad), de esta forma se estaría destruyendo empleo (efecto de desplazamiento o destrucción de empleo). Paralelamente, el aumento de la eficiencia del trabajo (y de los otros factores) causaría una reducción en el coste de producción unitario que mediante diversos mecanismos de compensación podría dar lugar a un incremento de la demanda y del empleo. En principio, existen cinco mecanismos indirectos asociados a la pérdida de empleo ocasionado por la introducción de nuevos procesos. Según los MDC, los efectos se coordinan en el tiempo y acaban desembocando automáticamente en un proceso de creación de puestos de trabajo (Antonucci \& Pianta, 2002).

Los tres primeros mecanismos de compensación (gráfico 2) se refieren a la utilización de los fondos librados debido a la reducción de costos generado por las nuevas innovaciones de proceso. Las ganancias en eficiencia productiva pueden dedicarse bien a una reducción de los precios, a mejorar el nivel salarial de los trabajadores o se puede desviar a los beneficios de los empresarios. La idea detrás del mecanismo de compensación basado en la reducción de precios es el siguiente. Al introducirse una innovación de proceso, la eficiencia del proceso productivo aumenta debido a una mejora de la productividad laboral. Tal reducción de costos implica, en un mercado competitivo, una disminución de los precios estimulando la demanda, que a su vez genera una mayor producción y empleo (Ley de Say). Sin embargo, existen ciertas críticas a este mecanismo. En primer lugar, la pérdida de empleo generada por la innovación también crea menor poder de compra de los trabajadores, que perdieron el empleo, lo que provoca una disminución de la demanda (Mill, 1976: 97). Esto significa que la demanda generada por la disminución del precio tiene que neutralizar o superar la menor demanda debido a la pérdida del empleo inicial.

Por otro lado, Vivarelli (2012) menciona tres requisitos que se deben de cumplir de forma simultánea para que el mecanismo de compensación vía precios ocurra. Primero, una alta elasticidad precio-demanda respecto a los bienes y servicios implicados; segundo, se requiere que dichos productos tengan un peso importante en la canasta de consumo de los trabajadores y tercero, se necesita una estructura de mercado competitivo, no monopólica ni oligopólica, para asegurar una reducción de los precios. 
Gráfico 2. El proceso económico detrás de algunos mecanismos de compensación

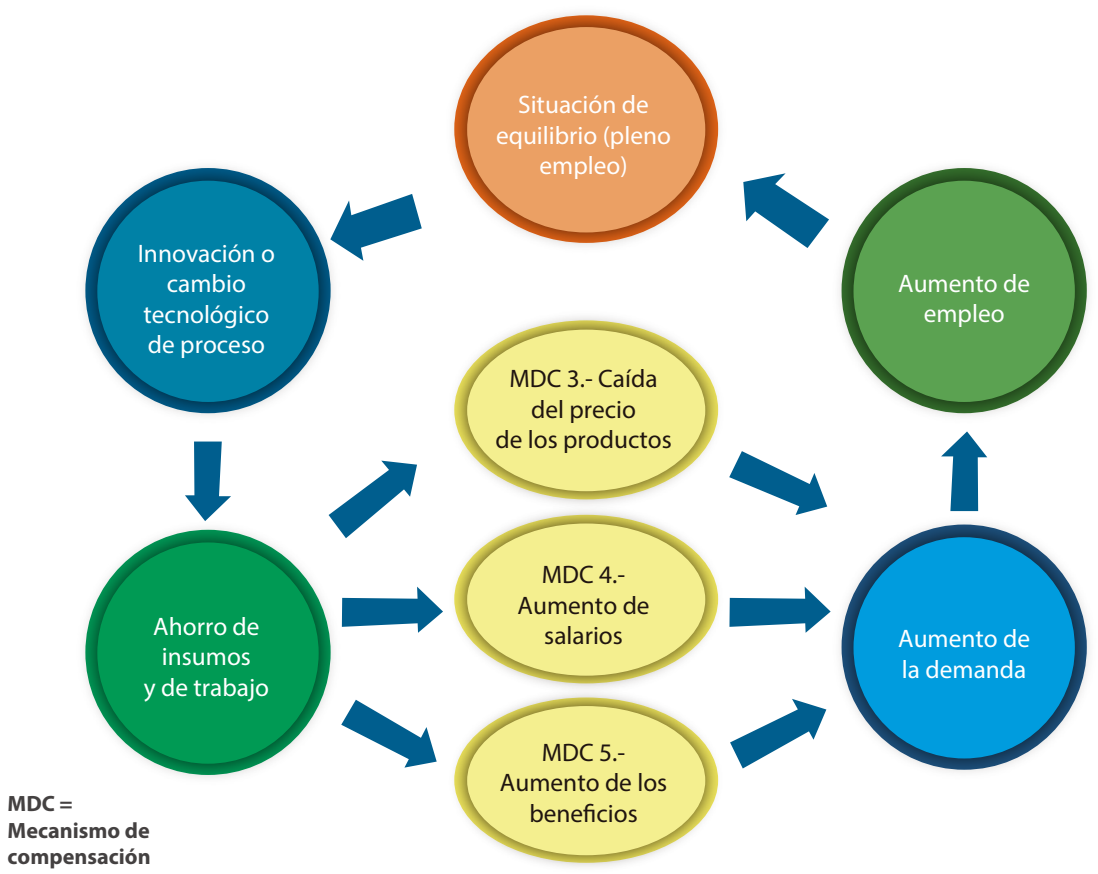

Fuente: Elaboración propia con base en Heijs et al. (2016).

Es importante mencionar que el ajuste del mercado no es instantáneo, por lo que el efecto entre el costo y el precio podría requerir cierto tiempo para que las ganancias de eficiencia y los menores costos puedan ser desviados a los beneficios de las empresas creando un segundo mecanismo de compensación. En el marco neoclásico los beneficios extraordinarios temporales se transforman automáticamente en nuevas inversiones que estimulan la demanda y la producción, compensando la perdida inicial de empleo parcialmente. Sin embargo, en la realidad económica, este efecto no ocurre automáticamente porque depende de las perspectivas futuras de los mercados, de los beneficios esperados y de los intereses personales o el "espíritu animal" por parte de los que podría retrasar el traslado de los beneficios adicionales a la inversión efectiva (Pasinetti, 1981; Freeman \& Soete, 1987; Vivarelli, 1995; Pianta, 2005).

Una tercera forma de utilizar el ahorro de costos sería un aumento de los sueldos de los trabajadores no expulsados. Este mecanismo de compensación se basa en la idea que un aumento salarial afectaría positivamente al consumo de los trabajadores, estimulando a su vez la demanda y la producción, por lo que se permite recuperar, parcialmente, 
la pérdida de empleo inicial. Como en los otros dos mecanismos, este argumento podría funcionar en un mercado de competencia perfecta que propone la corriente neoclásica. Sin embargo, esta corriente de pensamiento no toma en cuenta los periodos de auge y de crisis o el funcionamiento del mercado de trabajo en niveles altos de desempleo. El aumento salarial pudo ocurrir en la época dorada de la economía de los países más avanzados, desde los finales de 1950 hasta principios de 1970, siendo el único periodo, relativamente largo, de pleno empleo en estos países. En estos años el poder de los sindicatos era lo suficientemente alto para poder negociar un incremento de los salarios. Actualmente, la fuerza laboral está más fragmentada, con una oferta de trabajo amplia y altos niveles desempleo en comparación con los años de postguerra. Lo que conlleva a una pérdida del poder de negociación por parte de los sindicatos. Por lo tanto, este mecanismo ha sido fuertemente debilitado en el contexto institucional del desempleo actual (Vivarelli, 2012).

Un cuarto mecanismo de compensación está basado en el sector de máquinas y herramientas como sector emergente. Aunque, como resultado del progreso técnico, la introducción de nuevas máquinas implica un desplazamiento de la mano de obra, también se requieren obreros para fabricar las máquinas y para su distribución y mantenimiento. Lo que podría compensar parcialmente la pérdida inicial del empleo generado por las nuevas máquinas. Sin embargo, tal efecto compensatorio sería muy limitado, ya que el número de horas empleadas a fabricar las máquinas debe ser claramente menor al ahorro de empleo en los sectores usuarios de maquinarias y herramientas (Marx, 1969: 552). Si esto no fuera así, el costo de la introducción de las máquinas y herramientas sería mayor que la reducción del costo laboral, y ningún sector lo haría. Un segundo argumento en contra de este mecanismo se basa en las ideas de Marx (1867), quien subraya que, en el mediano y largo plazo, las tecnologías que ahorran mano de obra serán también introducidas en el sector de máquinas y herramientas. Siendo esta la realidad actual. Por otro lado, la introducción de nuevas máquinas puede deberse a una ampliación o a la sustitución de máquinas obsoletas, siendo este último el más frecuente. El impacto en términos de empleo en ambos casos es muy diferente. En el caso de sustituir las máquinas obsoletas no hay ningún efecto de compensación (Vivarelli, 2012; Freeman et al., 1982) y, de hecho, muchas veces la nueva maquinaria requiere menos mano de obra que la remplazada por lo que se pierden puestos de trabajo.

Un quinto mecanismo que podría amortizar la destrucción del empleo generado por la innovación de proceso es el efecto macroeconómico sobre los salarios (Vivarelli, 2007). El desempleo resultante de una mayor 
eficiencia y productividad implicaría una reducción de los salarios y, a su vez, la disminución del costo laboral induce, desde una perspectiva neoclásica, a los empresarios a orientar sus inversiones a tecnologías más intensivas en trabajo y, como consecuencia, contrataría más empleados (Wicksell, 1961: 137; Hicks, 1932:56; Pigou, 1933: 256). Este mecanismo puede ser criticado debido a las incongruencias teóricas y prácticas. Primero, una vez que la empresa ha comprado la nueva maquinaria es difícil revertir su uso y sustituirla por mano de obra (Dosi \& Nelson, 2010); segundo, se asume una perfecta sustitución de factores y, por lo tanto. se requiere una demanda homogénea en términos de capital humano, lo cual no es el caso (Schumpeter, 1939). Además, contrario al argumento teórico, los estudios empíricos no ofrecen evidencia en favor de la ausencia de la sustitución de factores (Vivarelli, 2012, Calvino \& Virgillito, 2018).

Además de los cinco mecanismos relacionados con la innovación de proceso también se puede identificar los efectos contradictorios de la innovación de producto sobre el empleo. En la literatura se suelen mencionar tres posibles consecuencias de las innovaciones de producto respecto a la cantidad del empleo (Peters et al., 2017; Vivarelli, 2014; Calvino \& Virgillito, 2018). En primer lugar, la introducción de productos totalmente nuevos en el mercado podría generar una nueva demanda lo que implicaría efecto positivo directo sobre el empleo. En segundo lugar, en el caso que se tratara de nuevos productos que sustituyeran o dejaran obsoletos a productos antiguos el efecto sobre el empleo sería indeterminado.

Según Schumpeter, el progreso tecnológico es un proceso de destrucción creativa, donde las innovaciones dejan obsoletos (sustituyen) bienes existentes, por lo que la creación de empleo en nuevos productos va aparejada con la destrucción de empleo en los productos obsoletos $\mathrm{y} / \mathrm{o}$ sustituidos, el efecto de sustitución, y el impacto neto en términos de puestos de trabajo no está claro (Katsoulacos, 1986; Pianta, 2000). El efecto neto, en este caso, dependería de si los nuevos productos se producen de forma más eficiente o no en comparación con los productos sustituidos. Si los nuevos productos son producidos más (menos) eficientemente que los viejos productos, estos requieren menos (más) insumos para una producción dada. Esto debilita (fortalece) el efecto positivo de la demanda y por lo tanto la creación o destrucción del empleo, efecto de productividad de la innovación de producto. Un tercer tipo de efecto, indirecto, de la demanda de nuevos productos se generaría si los nuevos productos en vez de sustituir a productos antiguos sean complementarios. En este caso podría estimular la demanda de los viejos y, por lo tanto, aumentar el empleo. 
Cabe mencionar que la teoría neoclásica sugiere que los efectos de compensación superan los efectos desplazamiento, de manera que las innovaciones (tanto de producto como de proceso) tengan consecuencias positivas sobre el empleo. En un mundo perfectamente competitivo donde cualquier oferta crea su propia demanda (Ley de Say), no sería posible que existiera desempleo de forma involuntaria, siempre que el mercado funcione con plena libertad. Según los neoclásicos, el cambio tecnológico toma parte en este proceso de auto-ajuste, donde el desempleo tecnológico sería una excepción a corto plazo en forma de desempleo friccional (Vivarelli, 2007), pero los tres mecanismos basados en la demanda e inversiones (aumento de beneficios, aumento de los salarios o la caída de los precios) que, según los supuestos de la teoría neoclásica sobre el mercado y la competitividad perfecta, deberían funcionar de forma automática entran en conflicto con la realidad económica donde su funcionamiento es muy limitado. De hecho, un amplio conjunto de factores determinará la magnitud del efecto neto de las innovaciones sobre el empleo (Pianta, 2005; Heijs et al., 2016, Calvino \& Virgillito, 2018) que podría ser tanto positivo como negativo.

Un primer aspecto que afectaría al efecto neto de los MDC sería la elasticidad renta-precio y, relacionado con este aspecto, el nivel de saturación de los mercados. Ambos aspectos dependen a su vez del PIB per cápita, la situación económica del país (crisis vs bonanza), el tipo de bienes (ej. bienes inferiores versus bienes de lujo), entre otros. En realidad, muchos nuevos productos compiten para una cuota del mercado cuya demanda es estable y saturada (ej. refrescos, moda, coches) donde una reducción de precios no genera un aumento en la demanda total y por ello del empleo. Igualmente, la introducción de nuevos productos en un mercado saturado solo puede realizarse para disminuir la cuota del mercado de otros productos (business stealing), por lo que el efecto sobre el empleo queda de nuevo en entredicho. En estos casos el desempleo generado por la innovación de proceso deja de ser un problema temporal y se convierte en un problema real y de largo plazo.

Otra faceta que influye sobre el efecto neto es la relevancia de la innovación para el sector productivo (innovaciones radicales o incremental; claves o marginales; productos intensivos en trabajo versus capital). Un tercer tipo de restricciones que afectan a los MDC sería el contexto institucional y legal de mercado de trabajo que afecta al poder de negociación de los trabajadores versus empresarios, como podrían ser el nivel de desempleo, el poder de sindicatos, el marco legal, en combinación con los "espíritus animales" y la reacción racional o emocional por parte del conjunto 
de los agentes económicos (empresarios y trabajadores). Un último aspecto para destacar que influye sobre el empleo neto es, como ya se indicó, la estructura del mercado especifico, especialmente, el nivel de competitividad y la existencia de productos sustitutivos.

Los estudios empíricos al respecto distinguen dos tipos de enfoques que tratan de analizar el efecto de la innovación en el empleo. En primer lugar, está el modelo empírico propuesto por Van Reenen (1997), y adaptado por Bogliacino \& Vivarelli (2012 y 2014), Pianta (2005), que conceptualiza la innovación con base en el esfuerzo innovador en Gasto en Innovación y Desarrollo (GID). Los autores de este enfoque encuentran un efecto positivo de esta variable en el empleo. Un segundo modelo, propuesto por Harrison et al. (2008 y 2014), utiliza dos indicadores de output de innovación distinguiendo entre el efecto de la innovación de producto y proceso en el empleo. Los estudios empíricos que aplican este modelo encuentran un efecto positivo de la innovación de producto en el empleo, mientras que en el caso de la innovación de proceso no se encuentran resultados contundentes (la mayoría de los estudios que confirman el efecto negativo de ésta variable sobre el empleo pero para algunos estudios el coeficiente resulta estadísticamente no significativo). ${ }^{7}$

\section{Efectos cualitativos de la innovación sobre el empleo: El cambio tecnológico sesgador de habilidades y rutinas}

Los mecanismos revisados anteriormente solo analizan el efecto sobre el empleo bajo un enfoque cuantitativo. Asumen el supuesto neoclásico que sostiene que el mercado de trabajo es estático, mientras que la evidencia empírica muestra que es dinámico, ya que está cambiando constantemente, creando y destruyendo tipos de trabajos, por lo que los efectos de la innovación en el empleo, no solo son en términos de números, sino el cambio tecnológico afecta al tipo de empleo, ya sea por habilidades, conocimientos o rutinas.

La literatura que analiza este fenómeno distingue dos tipos de trabajadores: calificados y no calificados. Welch (1970) destaca diversas razones que explican la mayor demanda de los trabajadores calificados y la necesidad de un mayor nivel de conocimientos. Una primera causa es el cambio estructural en la composición del sector productivo, donde los sectores que requieren trabajadores altamente calificados, siendo las industrias de alta tecnología, crezcan más rápido que los sectores tradicionales basados 
en trabajadores no calificados. En los países más avanzados, las industrias emergentes, que son las más intensivas en habilidades y conocimientos, crecen más rápido que las industrias tradicionales, de baja tecnología, ya que los productos de alta tecnología tienen una mayor elasticidad ingreso-demanda.

El aumento del bienestar en las décadas del 50 y $60^{8}$ generó un cambio en la composición del consumo y un cambio estructural del sistema productivo, ya que los trabajadores con un mayor poder adquisitivo requieren productos en un mayor nivel de prestaciones, calidad y seguridad, es decir, demandan más productos innovadores (Porter, 1993). Además, los mercados de los sectores de baja tecnología están relativamente saturados sin potencial de crecimiento. Una segunda razón de una mayor necesidad de obreros calificados mencionados por Welch (1970) es el aumento de intensidad de capital por trabajador. El incremento en el uso de factores no intensivos en mano de obra genera -ceteris paribus- un sesgo positivo en la demanda de trabajo calificado. En esta época, la inversión en capital sustituía sobre todo a los trabajadores bajamente calificados que realizan actividades rutinarias simples. El cambio tecnológico, además de expulsar empleo poco calificado, aumentaría, sobre todo, la productividad de los obreros calificados, desviando las inversiones hacia este tipo de actividades (Welch, 1970, Violante, 2008).

Para analizar el efecto de la innovación sobre el empleo en términos cualitativos en el mundo real, se ha introducido el concepto "Cambio Tecnológico Sesgador de Habilidades"(SBTC). ${ }^{9}$ Este concepto, mencionado por Nelson \& Phelps (1966), Griliches (1969) y Welch (1970), implica que la introducción de nuevas tecnologías requiere trabajadores con nuevas capacidades y habilidades distintas a los de las tecnologías anteriores. La hipótesis SBTC sugiere que las nuevas tecnologías y las habilidades requeridas son intrínsecamente complementarias al cambio tecnológico, lo que favorece contratar trabajadores calificados sobre los no calificados debido al aumento relativo de la productividad de los obreros calificados (Violante, 2008, Vivarelli, 2014).

En realidad, la literatura sobre el impacto del progreso tecnológico en la calidad del empleo, en términos de trabajo calificado y no calificado, recoge dos tipos de debates complementarios. Al inicio del siglo XX autores como Autor et al. (2003) y Goos et al. (2014) reformulan el

8 Debido a la mejor continua de la productividad a base de innovaciones de proceso.

9 Por sus siglas en inglés.

Teuken Bidikay Vol. 11 № 16 (Medellín, Colombia) Ene-Jun 2020. ISSN: 2215-8405 - e-ISSN 2619-1822. Pp. 55-89 
concepto del SBTC, argumentando que no se trata tanto de sesgo basado en las habilidades, sino que el cambio tecnológico sesgaría, sobre todo, la demanda del empleo según la índole rutinaria de los puestos de trabajo "Hipótesis de Cambio Tecnológico Sesgador de Rutinas" (RBTC). Esta hipótesis (Autor et al., 2008; Goos et al., 2014; Jaimovich \& Siu, 2012; Ford, 2015) pone el énfasis no tanto en el nivel de educativo y los conocimientos cognitivos sino en la previsibilidad o predictibilidad de las actividades, rutinarias versus no rutinarias.

El progreso tecnológico actual, basado en la IA, afecta especialmente a los trabajadores de calificación intermedia con puestos de trabajos que requieren una gran capacidad analítica basada en una gran cantidad de información compleja y enrevesada, pero cuya respuesta correcta es predecible y codificable y los conocimientos tácitos tienen un papel menor. Como indica Autor et al. (2003), estas tareas requieren de la repetición metódica de un procedimiento fijo, por lo que se pueden especificarlas detalladamente con instrucciones programadas y realizadas por máquinas. Según Brynjolfsson et al. (2017), existen diversos trabajos donde el tiempo necesario de la toma de decisiones por parte de los ordenadores es menor que el de los seres humanos y con un margen de error menor. ${ }^{10}$ La tecnología y, especialmente la IA, están reduciendo el tiempo de procesamiento de la información, la precisión y el número de errores humanos al respecto.

Aparentemente, la nueva revolución tecnológica afecta especialmente el empleo de "cuello blanco" de nivel intermedio, siendo los más susceptibles de ser sustituidos por las máquinas de IA (Webb 2019; Muro et al., 2019). Los trabajadores de un nivel profesional estarán cuatro veces más amenazados a la IA que los trabajadores con un grado de la enseñanza secundaria (Muro et al., 2019), por lo que en la llamada economía del conocimiento el grado universitario o secundario comienza a perder su distintivo de distinción (Brown et al., 2004). Esto implica que, "adquirir más educación y habilidades no necesariamente ofrece protección contra la automatización del trabajo futuro" (Ford, 2015: 111).

Dorn et al. (2016) profundizan en la misma idea y apuntan al impacto desigual sobre las tareas que realizan los trabajadores: CambioTecnológico Sesgador de Tareas (TBTC). ${ }^{11}$ La hipótesis de TBTC asume que la innovación 
sustituye aquellas actividades que siguen procedimientos bien definidos y previsibles, y considera cuatro tipos de trabajadores a base de las tareas que realizan (tabla 1) distinguiendo entre tareas rutinarias y no rutinarias y en cada una de estas dos opciones se distingue entre tareas manuales versus analíticas o abstractas ${ }^{12}$.

De acuerdo con la hipótesis de TBTC, aquellos trabajadores que realizan actividades rutinarias, no importando si son procesamientos analíticos o manuales, son susceptibles a ser sustituidos por la introducción de las nuevas tecnologías. Por otro lado, TBTC reconocen dos tipos de tareas no-rutinarias. La hipótesis TBTC clasifica como tareas abstractas aquellas que realizan actividades que resuelven problemas, requieren de creatividad o liderazgo de manejo, siendo las tareas no rutinarias basadas en procesamiento analítico. Estas actividades requieren trabajadores con alto nivel de conocimientos tácitos y una educación universitaria. El efecto de la innovación en este tipo de trabajadores generalmente es complementario, ya que muchas ocupaciones abstractas intensivas en conocimientos tácitos se volvieron más productivas cuando las computadoras permiten un procesamiento, almacenamiento y transmisión de datos más barato y rápido (Dorn, 2016). Es decir, las nuevas tecnologías permiten automatizar las tareas rutinarias de este tipo de trabajadores aumentando su productividad.

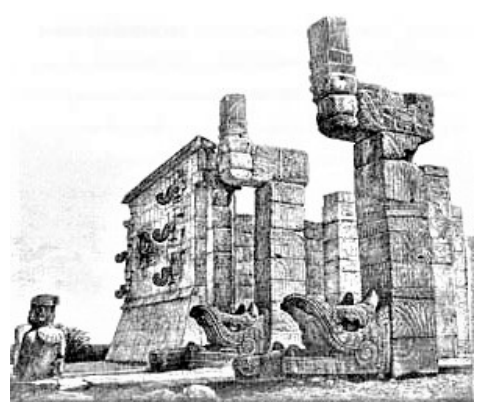

12 La diferencia entre RBTC y TBTC es más bien gradual y radica en que la segunda se centra en el tipo de actividades y/o tareas que pueden ser remplazadas por la tecnología, mientras que la primera, solo habla más bien de tareas rutinarias versus no rutinarias, sin entrar a detalle en el tipo de tareas (actividades) que llevan a cabo los trabajadores y el efecto de la tecnología sobre éstas. Es decir, donde las actividades rutinarias de los trabajadores se pueden automatizar, mientras que solo una parte de las actividades no rutinarias se automatizaría, siendo la tecnología complementaria con los trabajadores que desarrollan dichas actividades. 
Tabla 1. El impacto de la Innovación en cuatro categorías de tareas de trabajo

\begin{tabular}{|c|c|c|}
\hline \multicolumn{2}{|r|}{ Tareas rutinarias } & Tareas no rutinarias \\
\hline \multicolumn{3}{|c|}{ Procesamiento Analítico e Interactivo } \\
\hline \multirow{5}{*}{ Ejemplos } & * Registro de cuentas. & $\begin{array}{l}\text { *Formulación y prueba } \\
\text { de hipótesis. }\end{array}$ \\
\hline & *Cálculos. & * Diagnósticos médicos. \\
\hline & $\begin{array}{l}\text { *Servicios de los } \\
\text { consumidores repetitivos } \\
\text { (banquero). }\end{array}$ & *Escritos legales. \\
\hline & & *Persuasión y ventas. \\
\hline & & *Gestionar otras actividades. \\
\hline $\begin{array}{c}\text { Impacto } \\
\text { de la Innovación }\end{array}$ & Sustitución sustancial. & $\begin{array}{l}\text { Fuertemente } \\
\text { complementarias. }\end{array}$ \\
\hline \multicolumn{3}{|c|}{ Procesamiento Manual } \\
\hline \multirow{2}{*}{ Ejemplos } & $\begin{array}{l}\text { *Colección } \\
\text { y almacenamiento. }\end{array}$ & * Servicios de limpieza. \\
\hline & $\begin{array}{l}\text { *Proceso de montaje } \\
\text { repetitivo. }\end{array}$ & * Conducción de camiones. \\
\hline $\begin{array}{l}\text { Impacto de la } \\
\text { Innovación }\end{array}$ & Sustitución sustancial. & $\begin{array}{l}\text { Oportunidades limitadas } \\
\text { para la sustitución o } \\
\text { complementariedad. }\end{array}$ \\
\hline
\end{tabular}

Fuente: Elaboración propia con base en Autor et al. (2003).

EI TBTC define como un cuarto tipo de tarea las actividades no rutinarias y manuales, siendo aquellas las que se caracterizan por movimientos específicos, de reconocimiento visual y de comunicación verbal (como peluqueros, reparaciones, cuidado de niños). Siendo trabajos difíciles de sustituir por máquinas debido a que no siguen reglas o patrones homogéneas codificables. Aunque, contrario a los trabajadores de tareas abstractas, las nuevas tecnologías apenas ayudan aumentar su productividad (Dorn, 2016: 13).

Este proceso de automatización implica la polarización del empleo, donde las actividades de niveles intermedios son propensas a ser automatizados dejando una estructura laboral con mayor demanda de empleo en los extremos de la distribución por nivel de calidades. La hipótesis TBTC asume que la innovación no solo afecta el tipo de tareas que ejecutan los trabajadores, sino que también, indirectamente, afecta la distribución del ingreso y la desigualdad. Existe evidencia estadística que muestra que en países desarrollados la reducción de empleo intensivo en tareas rutinarias implica que el salario se concentrará en aquellos trabajadores que 
realizan tareas no rutinarias, tanto abstractas como manuales. Es decir, los trabajadores se concentrarán en mayor medida en los extractos de salarios, o muy altos o muy bajos del mercado laboral, a dicho fenómeno se le conoce como polarización del salario (Goos et al., (2014). Dorn (2016) menciona que en las últimas décadas los aumentos salariales fueron para aquellos que estaban en la parte superior e inferior de la distribución de ingresos y habilidades, y no para aquellos trabajadores localizados en la parte media.

La demanda de trabajo no rutinario frente al trabajo rutinario está relacionada directamente con el ciclo de vida de los productos. La etapa de la introducción de nuevos productos, inicialmente no estandarizados y con una baja demanda, implica la necesidad de procesos de producción intensivos en trabajo y con trabajadores altamente calificados. Una vez que el producto haya alcanzado éxito en el mercado y su diseño haya sido estandarizado, las empresas empiezan a introducir innovaciones de proceso que ahorran mano de obra para simplificar los procesos de producción. En las etapas del crecimiento y de la madurez del producto, la introducción de la innovación de proceso implica sustituir trabajadores calificados por no calificados. Por ejemplo, inicialmente el sector de las TIC creaba un aumento de personal calificado debido a la naturaleza y necesidades del trabajo (Berman et al., 1998 and Falk \& Biagi, 2017); sin embargo, la posterior estandarización de las nuevas tecnologías durante la fase de crecimiento en el ciclo de vida del producto y la familiarización de los usuarios con los softwares han reducido la demanda de trabajadores calificados (Tether et al., 2005).

Con base en la evidencia empírica ${ }^{13}$ sobre RBTC/SBTC/TBTC se puede indicar que el cambio tecnológico favorece a los trabajadores calificados, y desfavorece a los no calificados, remplazando tareas previamente realizadas por estos últimos, lo que aumenta la desigualdad.

\section{Progreso tecnológico, bienestar social y reconciliación familiar}

En la sección anterior se han mencionado el efecto de la innovación sobre la calidad de empleo en términos de los puestos de trabajo y de las tareas realizadas por los trabajadores. Otro aspecto importante con respecto a la calidad de empleo y bienestar social es la reconciliación entre la vida privada y laboral. Según un informe de la Oficina Internacional de Trabajo (OIT) (Messenger, 2018), existen dos aspectos que influyen directamente 
sobre tal reconciliación: el número total de horas trabajadas y la creciente flexibilidad del horario laboral. Respecto al primer aspecto se observa una bifurcación del tiempo de trabajo, donde la fuerza laboral del mundo se divide entre segmentos que viven en países menos desarrollados y experimentanjornadaslaborales y horas excesivas de trabajo; mientras que los trabajadores de los países avanzados cuentan con jornadas laborales más reducidas (Messenger, 2018). En los países avanzados se observa, en cierto modo, esta bifurcación donde existe un grupo de trabajadores muy calificados con una semana laboral mucho más reducidos y que se jubilan relativamente jóvenes y un grupo de trabajadores muy mal pagados con un horario laboral que superan las 50 horas semanales. Los estudios del ILO estiman que en los países más avanzados un 10\% de los trabajadores superan esta barrera, mientras que en los países en desarrollo casi una tercera parte de los trabajadores superan las 50 horas. En el caso de los países latinoamericanos este porcentaje llega al $22 \%$ y en Méjico está en un 29\% (OCDE; 2019).

Además del número de horas trabajadas, la calidad de vida depende también de la flexibilidad laboral, siendo un aspecto alterado por las nuevas tecnologías. Según Messenger (2018), las TIC han generado algunos cambios importantes en la situación laboral con consecuencias no deseadas respecto a los intereses de los trabajadores. Las TIC han borrado la frontera entre el tiempo dedicado a la vida profesional y el espacio de tiempo disponible para la vida privada. Las aplicaciones de internet que permiten una flexibilidad laboral excesiva, donde se dividen las tareas en pequeños paquetes que podrían ser asignadas a distintos trabajadores (como en el caso de Amazon, Uber, Didi Food). Tal forma de organización laboral implica jornadas laborales muy irregulares y poco predecibles, especialmente, en el caso de los llamados falsos autónomos o los trabajadores para empresas y trabajo temporal (ETT). Un problema que existe tanto en los países en desarrollo como en los países más avanzados. No siempre hay suficiente control que evita unas jornadas de trabajo excesivamente largas ni tampoco garantías en contra de los mini Jobs regulando unos contratos con unas horas mínimas de trabajo.

No se puede responsabilizar solo a las nuevas tecnologías o a los empresarios con pocos escrúpulos, aunque se requieren las TIC para organizar el trabajo de esta forma, la frontera de lo permitido y la protección a los trabajadores depende, en gran parte, de la regulación laboral. Por lo que el ILO propone políticas que deben regular un equilibro en el horario de los trabadores (que garanticen períodos mínimos de descanso y vacaciones pagadas) y que integran los intereses desde un punto empresarial y la alineación del trabajo con la vida privada del empleado. 


\section{El empleo y el modelo capitalista del consumo en masa y la obsolescencia programada}

Un análisis sistémico de las relaciones entre empleo, innovación, sostenibilidad y el bienestar en el modelo económico capitalista moderno ofrece un análisis bastante pesimista y desmonta parte del argumento a favor del mercado libre. Los autores neoclásicos defienden la no intervención estatal en la economía, argumentando la supremacía del libre mercado, cuya mano invisible generaría la asignación más eficiente de los factores productivos, pero el modelo capitalista moderno se destaca por un sistema poco eficiente en la utilización de materias primas, factores de capital y trabajo.

La asignación eficiente de los recursos debería implicar el uso de la capacidad potencial de nuevas tecnologías para conseguir productos casi indestructibles, pero, paradójicamente, el modelo capitalista actual, por lo menos en los países ricos, se basa en la ineficiencia programada del consumo en masa y excesivo, mediante la creación de un mercado cautivo que sigue comprando una y otra vez el mismo producto o donde los consumidores adquieren muchos más productos de los que realmente necesitan. Una de las formas de mantener el mercado cautivo y los beneficios a largo plazo y con un efecto colateral pero importante en forma de creación de empleo o, más bien, que origina el mantenimiento del empleo existente, es la estrategia empresarial conocida como obsolescencia programada. Esta estrategia ha sido utilizada de forma continua, por lo menos desde los principios del siglo XX (London, 1932; Gregory, 1947), e incluso ha sido defendido como una forma de conseguir la recuperación del empleo después de la crisis de los años 20 del siglo pasado (London, 1932).

La obsolescencia programada como estrategia de las empresas para mantener sus negocios y beneficios a largo plazo se basa en la sociedad de consumo, donde de forma premeditada el fabricante o las empresas diseñan los productos (o servicios) de tal forma que se acorta deliberadamente su vida útil, asegurándose que el producto se torne obsoleto en términos técnicos, de utilidad o a base de marketing. Asegurando que los productos se rompan con facilidad o dejen de funcionar a partir de un cierto momento para que el consumidor siga comprando el mismo producto una y otra vez. El objetivo empresarial es mantener la demanda en el mercado vendiendo el mismo producto (o uno muy similar) varias veces. Es decir, las empresas aseguran unas ventas futuras diseñando sus productos de tal forma que su ciclo de vida sea muy corto, bien porque se estropean fácilmente o bien porque se quedan tecnológicamente obsoletos o socialmente anticuados. 
Aunque el objetivo empresarial es mantener sus negocios con los beneficios correspondientes, esta estrategia tiene dos efectos negativos colaterales importantes. Por un lado, genera unos residuos pocos salubres y la sobreexplotación de las materias primas y otros recursos naturales escasos. Teorías sobre la economía sustentable y la protección del medio ambiente lo han abordado de manera amplia (Cardoso et al., 2018). De hecho, por parte de la Unión Europea se están tomando medidas de políticas para limitar la obsolescencia programada (Maitre-Ekern, 2016).

La segunda consecuencia de la obsolescencia programada está relacionada con un efecto aparentemente positivo sobre la sustentación del empleo, pero tiene un efecto negativo sobre el bienestar. En realidad, el efecto positivo de la obsolescencia programa en el empleo es una argucia de mantener felices a los trabajadores mediante un salario que mejoraría su nivel de vida, pero en realidad se podría haber aprovechado el progreso tecnológico para producir productos de calidad y durables, para reducir así las horas de empleo necesarias, manteniendo el mismo nivel de bienestar social con menos presión sobre las materias primas y el medioambiente, creando una situación win-win. Todo el mundo podría haber mejorado su nivel de bienestar e incluso, teóricamente, los empresarios podrían haber mejorado su nivel de vida en términos de cantidad de bienes que tendrían potencialmente disponibles.

\section{Innovación y la distribución internacional del empleo y la riqueza}

En esta sección se debate muy brevemente algunos aspectos de la distribución de la riquezay el empleo generado por el progreso tecnológico y las implicaciones para los países en desarrollo, especialmente, de América Latina. Un primer aspecto es la distribución internacional del empleo en las cadenas de valor y según el ciclo de vida de los productos, describiendo las tendencias del último siglo. Seguido por una breve discusión respecto al potencial de la nueva revolución tecnológica ${ }^{14}$ sobre el retorno de la producción industrial a los países más avanzados.

Otro aspecto que se trata en esta sección es la desigualdad del crecimiento de la productividad en los países menos desarrollados, a base de avance tecnológico, y de la absorción de los beneficios correspondientes. Aparentemente, los países más avanzados absorben una parte relevante de las ganancias de la productividad creado por los países pobres. Tal 
debate se encuadra en la hipótesis de la relación de intercambio de bienes de Prebisch y Singer, y se relaciona con la falta la voluntad de erradicar la pobreza absoluta a pesar de que el cambio tecnológico permite que a nivel internacional se produzca más riqueza que nunca.

Los avances en las TIC entre 1960-2000 permitieron la fragmentación de la producción y un cambio profundo de la forma de comercializar $y$, sobre todo, de producir los productos. En este período se han visto alteradas las cadenas de valor global (CVG) ${ }^{15}$ donde la fabricación de un solo producto se realiza de forma fragmentada en distintos países del mundo, trasladando parte o el total del proceso productivo a países de salarios bajos. De hecho, la deslocalización implicó la pérdida de un tercio de la producción industrial europea en este período (Westkämper, 2014: 109). En otras palabras, el papel de la innovación y el poder económico de los países más avanzados ha tenido un efecto claro en la distribución internacional del trabajo con desigualdades en la calidad del empleo, tanto en términos de la necesidad de calificaciones avanzadas o el nivel de salarios (poder de compra) como en términos de la seguridad y salubridad del empleo.

Se puede añadir que la distribución internacional del empleo según su nivel de calidad, altamente calificado en los países avanzados y de baja calificación en los países en desarrollo, está directamente ligada al ciclo de vida del producto y a la trayectoria tecnológica típica del proceso de innovación. Los países más avanzados han podido aprovecharse de los países de bajos salarios para la producción de un amplio espectro de bienes estandarizados, especialmente los de consumo, que se encuentra al final del ciclo de vida del producto. La introducción de nuevos productos ${ }^{16}$ implica un proceso productivo intensivo en trabajo que requiere empleados altamente calificados y se beneficia de unos consumidores exigentes para producir el nuevo producto, asegurando su mejora continua mediante innovaciones incrementales. Además, en estos primeros compases del ciclo de vida del producto existe escasez de profesionales calificados, por lo que las empresas tienen que pagar un salario alto para atraer a los pocos profesionales existentes.

15 Es el conjunto de las personas y actividades involucradas en la producción de un bien o servicio a nivel global.

16 Productos "pre-paradigmáticos", considerados innovaciones radicales sin estándares dominantes claramente definidos. 
Una vez asentado el mercado, con una demanda (esperada) suficientemente grande, se inicia la fase de innovación de proceso de la actividad manufacturera con base en la estandarización del producto y sus componentes, la simplificación y estandarización de las tareas productivas y del aprendizaje por parte de los obreros, lo que permitiría la mecanización de su producción. Este proceso puede implicar a largo plazo la obsolescencia de las calificaciones de los trabajadores de los países avanzados, formados durante las primeras etapas del ciclo, o sus habilidades y destrezas se pueden conviertir en un bien común, por lo que ya no se pagaría un sobre precio (salarios altos) para su empleo. El proceso de estandarización y las ventajas de escala de los mercados crecientes incentivan a un proceso productivo intensivo en capital con trabajadores pocos calificados en países de salarios bajos.

La paradoja es que una vez que el PIB de un país dependa básicamente de la producción de bienes de bajo valor añadido habrá un freno al aumento de su nivel salarial, porque podría amenazar los puestos de trabajo de muchos obreros. Además, muchos de los países en esta situación son menos restrictivos en la aplicación de las normas de seguridad y salubridad con el objetivo de reducir costes.

La nueva revolución tecnológica basada en la aplicación de la IA a las TIC podría dar de nuevo un giro a la CVG, ya que la creciente capacidad productiva y de autoaprendizaje de la robotización, en combinación con la reducción de su coste, podría facilitar el retorno de la producción industrial a los países avanzados (De Backer et al., 2018). Además, las ventajas de los robots, no solo se limita a su mayor nivel de productividad, sino que también implica una mayor regularidad en términos de la calidad de los productos (incluido la reducción del número de errores humanos y productos imperfectos y costes para el control de calidad) y mayor nivel de seguridad y salubridad laboral. Las nuevas aplicaciones basadas en la IA y la existencia de robots cada vez más pequeños, más fáciles de mover o ajustar, permiten flexibilidad para cambiar las características de los productos a fabricar, lo que es importante para una mayor innovación de productos y exigencias de productos personalizados.

Todavía la mayoría de los robots no tienen la flexibilidad requerida y muchos de sus movimientos están planificados y programados previamente. Sin embargo, las máquinas serán más flexibles debido al progreso de la IA y la capacidad del autoaprendizaje y la autocorrección de los robots, lo que les permite realizar una gama más amplia de actividades complejas. Las máquinas serán más capaces de hacer muchas tareas con mayor precisión, más baratas y más rápidas, lo que las hace cada vez más 
rentables y accesibles. ${ }^{17}$ Como se mencionó en la introducción, se estima una pérdida potencial del empleo, entre el $40 \%$ y $50 \%$ de los puestos a nivel mundial (McKinsey, 2017; Frey \& Osborne, 2017). Otros estudios esperan efectos significativamente menores (OCDE, 2016A; Graetz \& Michaels, 2015; Acemoglu \& Restrepo, 2017). Aunque, como se indica, es todavía pronto para saber el efecto a largo plazo.

Otro aspecto importante es la distribución del beneficio de las ganancias en productividad entre países desarrollados y en vías de desarrollo. No es verdad que los países en desarrollo no mejoran la productividad de sus trabajadores y tal mejora debería aumentar el bienestar general, pero resulta que, sobre todo, los países más avanzados absorben las ganancias de los países económicamente menos avanzados. Como muestran Prebisch $(1949,1950)$ y Singer (1998), el aumento de la productividad en los países poco desarrollados, paradójicamente, no implica un aumento de los salarios de los trabajadores que produzcan más, sino que las ganancias de su productividad, especialmente en el sector de la agricultura, han sido absorbidas por los consumidores de los países ricos, donde se bajaron los precios de consumo de este tipo de productos.

Este desuso de las ganancias de la eficiencia se debe, según Prebisch, a los deterioros dela relación de intercambio. Los precios delasimportaciones de los países pobres crecen más rápido que los precios de sus exportaciones, porque los precios mundiales de los productos básicos (pocos o no elaborados) crecen menos que los precios de los productos industriales (de media y alta tecnología). Los países avanzados cobran aranceles mucho más altos ${ }^{18}$ sobre los productos elaborados o semielaborados. En resumen, los países en desarrollo tienen que exportar cada vez más cantidades de sus productos industriales estandarizados y agrícolas para financiar la importación de una igual cantidad de productos de los países ricos, perdiendo gran parte de las ganancias de productividad. Debido a la innovación de proceso y al aumento correspondiente de la productividad se podría sostener una reducción de la jornada laboral manteniendo la cantidad de bienes y servicios producidos a nivel internacional, tanto en términos absolutos, como per cápita.

17 EBCG (2015) estima que el costo de los robots disminuirá en un 20\% y su rendimiento mejorará en un 5\% anual durante la próxima década.

18 Como los aranceles progresivos que son cada vez más elevados a los productos que provienen de países pobres, según se avance en las etapas de elaboración. La progresividad arancelaria es uno de los obstáculos para los países en desarrollo que quieren establecer industrias de elaboración en vez de exportar sus bienes primarios. 
Actualmente, se producen más productos que nunca, por lo que, de acuerdo con la teoría, el nivel de bienestar medio en términos económicos, es decir, la cantidad de productos disponibles por persona debería aumentar. Lo que permitiría un nivel mínimo de bienestar más alto y debería facilitar una distribución de la riqueza mucho más equilibrada, pero, paradójicamente, se ha observado un incremento de la desigualdad dentro y entre países y se detecta una parte de la población a nivel mundial totalmente ajena al avance económico (gráfico 3). Todavía un 10\% de la población mundial vive en la pobreza extrema ${ }^{19}$ y casi la mitad vive con menos de $\$ 5,50$ al día y para Méjico este porcentaje es del 35\% (Banco Mundial, 2018). Esto implica que, a pesar del progreso tecnológico y el aumento de la productividad, no se ha conseguido erradicar la pobreza extrema ni la muerte infantil, que se sitúa en un promedio anual de 6 millones niños. ${ }^{20}$

Gráfico 3. Participación en el consumo mundial de los quintiles más ricos y más pobres de la población en el siglo XX

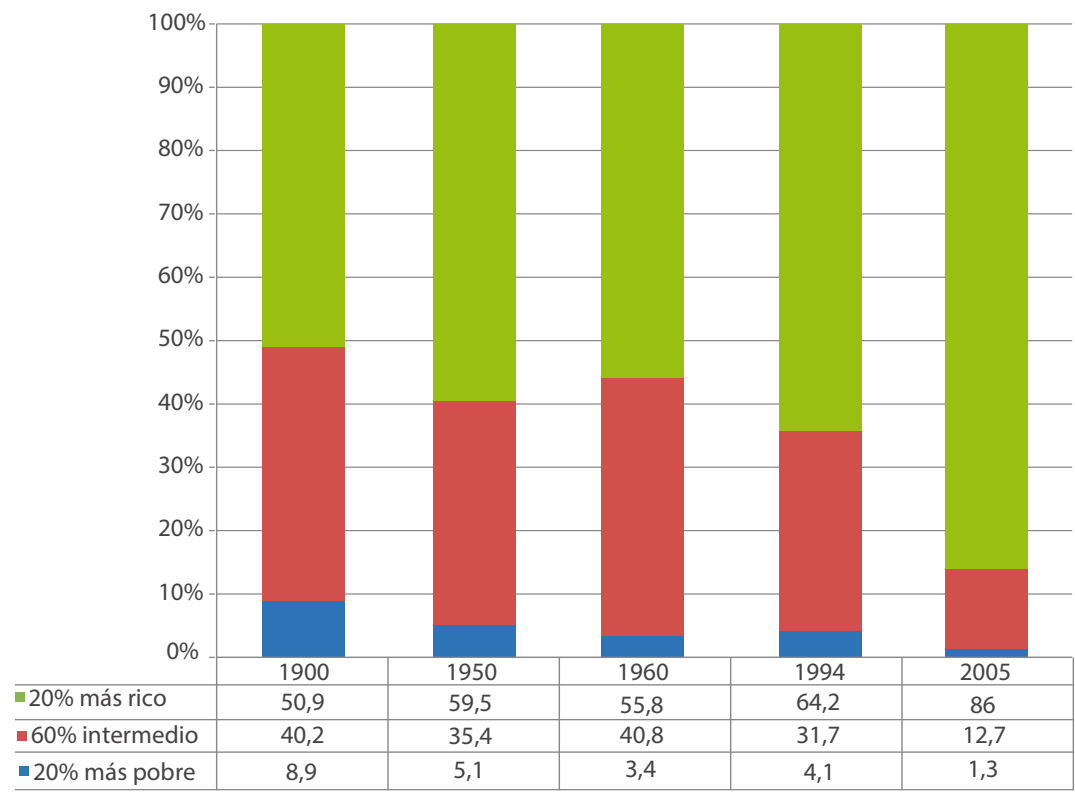

Fuente: Elaboración propia con base en datos de Roser y Ortiz (2020).

19 Con menos de $\$ 1,90$ al día en paridad de poder de compra.

20 Cada cinco segundos un niño muere, según las estimaciones de Naciones Unidas. 
El problema del efecto de la innovación en el empleo no es tanto el desempleo que crea sino la distribución, que cada vez es más desigual, donde las ganancias en la productividad han sido captadas por una minoría. El capitalismo ${ }^{21}$ y la industrialización como parte del proceso

de innovación han creado en los países ricos una sociedad de consumo (Akerlof \& Shiller, 2009). La se agudiza pobreza en los países menos desarrollados. Como se observa en el gráfico 3 el veinte por ciento de la población más rica del mundo derrocha el $86 \%$ del consumo, mientras que el $20 \%$ más pobre solamente realiza el $1 \%$ del consumo. Para Méjico estos porcentajes son respectivamente el 54,2\% y el 4,9\% en 2016 (The World Bank, 2019). Cabe destacar que hace algo más de un siglo estos porcentajes fueron respectivamente el $51 \%$ y el $9 \%$, lo que confirma la mayor concentración de la riqueza en un grupo pequeño de la población.

\section{Comentarios finales}

En esta sección final se retoman muy brevemente algunos conceptos y paradojas del efecto de la innovación en el empleo en términos socioeconómicos. Cuando se habla de los efectos de la innovación en el empleo se discuten implícitamente los efectos socioeconómicos y el nivel bienestar. La distribución de ganancias en productividad y eficiencia es muy desigual en un país como entre países. El problema no es tanto el hecho que la innovación genera una pérdida de empleo, sino los efectos que tiene la innovación en la composición del mercado laboral y las distintas formas de polarización mencionada en el texto.

La innovación no implica de forma irrevocable pobreza para los que pierden su empleo, ya que no se disminuye la producción total en el mundo, sino que la pobreza y desigualdad son el resultado de la mala distribución de la riqueza entre los habitantes de nuestro planeta donde no se asegura un nivel mínimo de bienestar accesible a todo el mundo. En este sentido, quizás, más importante que el pleno empleo, sería una distribución equitativa del trabajo y mejores niveles de vida y tiempo de ocio. La distribución de la riqueza debe basarse en la reducción de la jornada laboral y en la implementación de incentivos de recompensa al esfuerzo y capacidad laboral. 
Se debe superar el modelo económico actual de un capitalismo cada vez más salvaje con una protección decreciente de los derechos de los trabajadores debido a la emergencia de los mini-jobs, los falsos autónomos y el modelo de consumo en masa basado en la obsolescencia programada con el uso indiscriminado de las materias primas. Un problema derivado de la obsolescencia programa es el hecho de que el alto nivel de consumo en los países avanzados, que son los que acaparan los beneficios del proceso de innovación, están agotando los escasos recursos naturales (de energía, de materiales primas y del medio ambiente). El agotamiento de los recursos no ha sido para mantener el alto nivel de vida, sino que es consecuencia del modelo ineficiente de consumo en masa y de la obsolescencia programada que han intensificado el aumento de los precios de las materias primas, de los recursos naturales e incluso de los alimentos básicos, ${ }^{22}$ lo que de nuevo tiene un efecto negativo sobre el poder adquisitivo de la parte más pobre de la población. ${ }^{23}$

El desarrollo de los países pobres no es fácil en el contexto internacional actual. Los países más avanzados han podido solucionar muchos de los problemas del propio proceso de desarrollo y crecimiento económico, porque durante su industrialización tenían disponibles unos mercados internacionales "vírgenes" sin competidores y recursos naturales abundantes. Además, para solucionar los problemas de la explosión demográfica, ${ }^{24}$ los trabajadores, en los siglos XIX y XX, expulsados de su sistema productivo, debido a la industrialización, han emigrado en masa a los países menos desarrollados teniendo facilidades que no disponen los países en desarrollo o pobres actuales. El libre comercio en estos países tampoco fue tan libre como se pretende aparentar (Chang \& Salomón, 2004).

El cambio tecnológico, en combinación con el poder de los países avanzados y el modelo capitalista, ha permitido o impuesto el surgimiento de un mundo polarizado con base en un conjunto amplio de países con

22 La cantidad de alimentos per cápita desperdiciada por los consumidores es de 95 a $115 \mathrm{~kg} / a$ ño en Europa y América del Norte, mientras que esta cifra alcanza solo de 6 a $11 \mathrm{~kg} /$ año en el África subsahariana y Asia meridional y sudoriental (FAO, 2012).

23 Resulta que los paises avanzados compran de forma barata las materias primas de los países pobres que ayudan a convertirlos en bienes de consumo no demasiado duradero debido a la obsolescencia programada. $Y$ una vez que tiran los productos las materias primas se devuelvan a los países pobres en forma de basura, metales pesados y otros materiales tóxicos muy peligrosos para el medio ambiente (tomado de una referencia desconocida).

24 Siendo uno de los grandes problemas durante la industrialización (dejándose atrás la sociedad agraria). 
salarios bajos, largas jornadas laborales y altas tasas de desempleo que funcionan como la fábrica del mundo. Mientras en un grupo pequeño de países existe un menor nivel de desempleo. Siendo éstos los países más innovadores donde predominan los salarios altos, sobre todo, porque compensan su mayor productividad trabajando menos horas. En el futuro tampoco queda garantizada una mejora de la situación de los países menos desarrollados.

La nueva revolución tecnológica, basada en la robotización, la Inteligencia Artificial y las fotocopiadoras 3D, podría afectar, de manera negativa, a los países más débiles debido a un posible proceso de retorno de la producción industrial y manufacturera a los países más avanzados. Las nuevas tecnologías del futuro (la industria 4.0) implicarían una automatización, donde podría desaparecer más del 50\% del empleo industrial (McKinsey, 2017; Frey y Osborne 2017), lo que a su vez implica que el coste laboral es un aspecto cada vez menos importante para competir. Muchas de las actividades, que se producían en países de salarios bajos (subdesarrollados), podrían regresar a los países más avanzados (conceptualizado como backshoring), lo que hace que los países más desarrollados acaparen el aumento de la productividad generado por el cambio tecnológico. Aunque ya existe literatura que analiza conceptual y empíricamente el tema del retorno es pronto para evaluar las consecuencias a largo plazo de este proceso, pues estamos al principio de esta nueva revolución tecnológica, pero no cabe duda de que las previsiones al respecto no son del todo halagadoras para los países menos desarrollados.

Aunque, en este caso, Méjico podría tener una ventaja debido a su cercanía a EE UU, e incluso podría atraer parte de la producción que se retorna debido al concepto near-shoring. Méjico podría ser una localización de la producción que combina las ventajas de estar suficientemente cercana al mercado estadounidense y con ventajas de localización de un país de nivel intermedio de desarrollo.

En este artículo se han revisado diversas paradojas o contradicciones respecto al papel de la innovación para el desarrollo del mercado laboral en términos cuantitativos y cualitativos. No solo desde una perspectiva puramente económico, sino también analizando la polarización o distribución desigual de las ganancias en eficiencia y productividad de los trabajadores de los países menos desarrollados y el potencial de la tecnología para mejorar el bienestar social en este tipo de países. 


\section{Referencias bibliográficas}

1. ACEMOGLU, Daron. (2002). Technical change, inequality, and the labor market. Journal of economic literature, Vol. 40, № 1. Pp. 7-72. DOI: 10.1257/0022051026976

2. ACEMOGLU, Kamer Daron \& RESTREPO, Pascual. (2017). Robots and jobs: Evidence from US labor markets. NBER Working Paper, № 23285. Retrieved from http://www. nber.org/papers/w23285. Consultado 10.10.2019

3. AKERLOF, George Arthur \& SHILLER, Robert. (2009). How animal spirits destabilize economies. McKinsey Quarterly, Vol. 3. Pp. 127-135.

4. ANTONUCCI, Tommaso \& PIANTA, Mario. (2002). Employment effects of product and process innovation in Europe. International Review of Applied Economics, Vol. 16, No 3. Pp. 295-307. https://doi.org/10.1080/02692170210136127

5. ARNTZ, Melanie, GREGORY, Terry \& ZIERAHN, Ulrich. (2017). Revisiting the risk of automation. Economics Letters, Vol. 159. Pp.157-160. https://doi.org/10.1016/j. econlet.2017.07.001

6. AUTOR, David, KATZ, Lawrence \& KEARNEY, Melissa S. (2008). Trends in US wage inequality: Revising the revisionists. The Review of economics and statistics, Vol. 90, No 2. Pp. 300-323. https://doi.org/10.1162/rest.90.2.300

7. AUTOR, David, LEVY, Frank \& MURNANE, Richard J. (2003). The skill content of recent technological change: An empirical exploration. The Quarterly journal of economics, Vol. 118, № 4. Pp. 1279-1333. https://doi.org/10.1162/003355303322552801

8. BANCO MUNDIAL. (2018). Poverty and Shared Prosperity: Piecing Together the Poverty Puzzle (Washington, DC: Grupo Banco Mundial). Retrieved from: https://www. worldbank.org/en/publication/poverty-and-shared-prosperity

9. BERMAN, Eli BOUND, John \& MACHIN, Stephen. (1998). Implications of skill-biased technological change: international evidence. The quarterly journal of economics, Vol. 113, No4. Pp. 1245-1279. https://doi.org/10.1162/003355398555892

10. BOGLIACINO, Francesco \& VIVARELLI, Marco. (2012). The job creation effect of R\&D expenditures. Australian Economic Papers, Vol. 51, № 2, Pp. 96-113. https://doi. org/10.1111/j.1467-8454.2012.00425.x

11. BOGLIACINO, Francesco, PIVA, Mariacristina \& VIVARELLI, Marco. (2014). Technology and employment: The job creation effect of business R\&D. Rivista Internazionale di Scienze Sociali, Vol. 122, № 3. Pp 239-264. https://www.jstor.org/stable/43830213

12. BROWN, Phillip, HESKETH, Anthony \& WILLIAMS, Sara. (2004). The mismanagement of talent: Employability and jobs in the knowledge economy. $1^{\text {a }}$ ed. Oxford: Oxford University Press. 288 pp.

13. BRYNJOLFSSON, Erik, ROCK, Daniel \& SYVERSON, Chad. (2017). Artificial intelligence and the modern productivity paradox: A clash of expectations and statistics. National Bureau of Economic Research, № 24001. https://doi.org/10.3386/w24001

14. CALVINO, Flavio \& VIRGILLITO, Maria Enrica. (2018). The innovation-employment nexus: A critical survey of theory and empirics. Journal of Economic Surveys, Vol. 32, № 1. Pp. 83-117. https://doi.org/10.1111/joes.12190 
15. CARDOSO, Walter, SACOMANO, José Benito, CONTADOR, José Celso \& TELLES, Renato. (2018). Planned obsolescence or planned resource depletion? A sustainable approach. Journal of cleaner production, Vol. 195. Pp. 744-752. https://doi.org/10.1016/j. jclepro.2018.05.222

16. CHANG, Ha-Joon \& SALOMÓN, Mónica (2004). Retirar la escalera: la estrategia del desarrollo en perspectiva histórica. Madrid: Los libros de la Catarata. 247 pp.

17. DAVID, Paul. (1991), Behind the Diffusion Curve. Oxford: Westview Press. 150 pp.

18. DE BACKER, Koen, DESTEFANO, Timothy, MENON, Carlo \& SUH, Jung. (2018). Industrial robotics and the global organisation of production. OECD Science, Technology and Industry Working Papers, №. 03. Pp. 1-43. https://doi.org/10.1787/dd98ff58-en

19. DORN, David. (2016). The rise of the machines: How computers have changed work. UBS Center Public Paper Series 4, University of Zurich: UBS International Center of Economics in Society. Retrieved from: https://www.ubscenter.uzh.ch/de/publications/ public_papers.html. Consultado: 17.08.2019.

20. DOSI, Giovanni \& NELSON, Richard. (2010). Technical change and industrial dynamics as evolutionary processes (pp. 51-127). In: B. H. Hall \& N. Rosenberg (Eds.), Handbook of the Economics of Innovation. Amsterdam: North-Holland.

21. FALK, Martin \& BIAGI, Federico. (2017). Relative demand for highly skilled workers and use of different ICT technologies. Applied Economics, Vol. 49, № 9. Pp. 903-914. https://doi.org/10.1080/00036846.2016.1208357

22. FAO. (2012). Pérdidas y desperdicio de alimentos en el mundo. Alcance, causas y prevención. Roma. Disponible en Internet: http://www.fao.org/3/i2697s/i2697s00. htm. Consultado: 5.07.2019.

23. FORD, Martin. (2015). The rise of the robots: Technology and the threat of mass unemployment. $1^{\text {a }}$ ed. London: One world publications. $295 \mathrm{pp}$.

24. FREEMAN, Chris \& SOETE, Luc. (1987). Factor substitution and technical change (pp. 2187-2183). In: C. Freeman \& L. Soete (Eds.), Technical Change and Full Employment. Oxford: Basil Blackwell.

25. FREEMAN, Chris \& SOETE, Luc. (1994). Work for all or mass unemployment? Computarised technical change into the twenty-first century ( $\left.N^{\circ} 331.101 .5 \mathrm{FRE}\right) .193 \mathrm{pp}$.

26. FREEMAN, Chris, CLARK, John, \& SOETE, Luc. (1982), Unemployment and Technical Innovation: A study of long waves and economic development. London: Pinter. 252 pp.

27. FREY, Carl Benedikt \& OSBORNE, Michael. (2017). The Future of Employment: How Susceptible are Jobs to Computerisation? Technological Forecasting and Social Change, Vol. 114. Pp. 254-280. https://doi.org/10.1016/j.techfore.2016.08.019

28. GOOS, Maarten, MANNING, Alan, \& SALOMONS, Anna. (2014). Explaining job polarization: Routine-biased technological change and offshoring. American Economic Review, Vol. 104, № 8. Pp. 2509-2526. https://doi.org/10.1257/aer.104.8.2509

29. GRAETZ, Georg \& MICHAELS, Guy. (2015). Robots at Work. CEPR Discussion Paper No. DP10477. Retrieved from: https://ssrn.com/abstract=2575781. Consultado: 23.09.2019. 
30. GREGORY, Paul. (1947). A theory of purposeful obsolescence. Southern Economic Journal, Vol. 14, № 1. Pp. 24-45. https://doi.org/10.2307/1052870

31. GRILICHES, Zvi. (1969). Capital-skill complementarity. The Review of Economics and Statistics, Vol. 51, No 4. Pp. 465-468. https://doi.org/10.2307/1926439

32. HARRISON, Rupert, JAUMANDREU, Jordi, MAIRESSE, Jacques \& PETERS, Bettina. (2008). Does Innovation Stimulate Employment? A Firm-Level Analysis using Comparable Micro-Data from Four European Countries. NBERWorking Paper 14216.Cambridge MA: NBER. Retrieved from: http://www.nber.org/papers/w14216. Consultado: 23.09.2019.

33. HARRISON, Rupert, JAUMANDREU, Jordi, MAIRESSE, Jacques \& PETERS, Bettina. (2014). Does innovation stimulate employment? A firm-level analysis using comparable microdata from four European countries. International Journal of Industrial Organization, Vol. 35. Pp. 29-43. https://doi.org/10.1016/j.ijindorg.2014.06.001

34. HEIJS Joost, MARQUÉS, Gabriel y COVADONGA, María. (2016). El impacto de las innovaciones de producto y proceso sobre el empleo industrial. En: VERGARA, Delia Margarita. Innovación, salarios, y eficiencia productiva. Ciudad de Méjico (Méjico): Instituto de Investigaciones Económicas UNAM.

35. HEIJS, Joost, ARENAS, Díaz \& VERGARA, Delia Margarita. (2019). Impact of innovation on employment in quantitative terms: review of empirical literature based on microdata. Working Paper in MPRA. Retrieved from: https://mpra.ub.uni-muenchen. de/id/eprint/95326 Consultado: 12.10.2019.

36. HICKS, John Richard. (1932). The Theory of Wages. $1^{\text {a }}$ ed. London: Macmillan. 247 pp.

37. HOBSBAWM, Eric J. (1952). The Machine Breakers (pp. 57-70). In Past \& Present, № 1. Retrieved from: www.jstor.org/stable/649989

38. INTERNATIONAL LABOUR OFFICE (2018). Ensuring decent working time for the future: General Survey concerning working-time instruments. Report III (B), International Labour Conference, 107th Session, Geneva. 2018.

39. JAIMOVICH, Nir, SIU, Henry. (2012). The trend is the cycle: Job polarization and jobless recoveries (No. w18334). National Bureau of Economic Research.

40. JENKINS, David Trevor. (1994). The Textile Industries. In: Church, R.A. \& Wrigley, E.A. (Eds.), The Industrial Revolution in Britain. Oxford: Blackwell.

41. KATSOULACOS, Yannis S. (1986). The employment effect of technical change: A theoretical study of new technology and the labor market. London: University of Nebraska Press.

42. KEYNES, John M. (1930). Economic possibilities for our grandchildren. In Essays in persuasion. London: Palgrave Macmillan.

43. KEYNES, John M. (1936). The general theory of employment, interest and money. In The Collected Writings of John Maynard Keynes. $1^{\text {a }}$ ed. London: Macmillan.

44. LONDON, Bernard. (1932). Ending the depression through planned obsolescence. Madison: University of Wisconsin Press.

45. MACHIN, Stephen. (2001). The changing nature of labour demand in the new economy and skill biased technology change. Oxford Bulletin of Economics and Statistics, Vol. 63, № 1. Pp. 753-776. 
46. MADDISON, Angus. (2001).The World Economy: A Millennial Perspective, Development Centre Studies. Paris: OECD Publishing. https://doi.org/10.1787/9789264189980-en

47. MAITRE-EKERN, Eléonore \& DALHAMMAR, Carl. (2016). Regulating planned obsolescence: a review of legal approaches to increase product durability and reparability in Europe. Review of European, Comparative \& International Environmental Law, Vol. 25, №3. Pp. 378-394. https://doi.org/10.1111/reel.12182

48. MANSFIELD, Edwin. (1963). Intrafirm rates of diffusion of an innovation. The review of economics and statistics, Vol. 45, № 4. Pp. 348-359. https://doi.org/10.2307/1927919

49. MARX, Karl. (1867). El Capital. $1^{\text {a }}$ ed. Moscow: Foreign Languages Publishing House. 3183 pp.

50. MARX, Karl. (1969). Theories of Surplus Value. London: Lawrence \& Wishart. 1910 pp.

51. MCKINSEY GLOBAL INSTITUTE (2017). A future that works: Automation, employment and productivity, McKinsey Global Institute. Retrieved from: https://www. mckinsey.com/ /media/McKinsey/Featured\%20Insights/Digital\%20Disruption/ Harnessing\%20automation\%20for\%20a\%20future\%20that\%20works/MGI-A-futurethat-works_Full-report.ashx

52. MESSENGER, Jon. (2018). Working time and the future of work, Future of Work Research Paper Series. ILO №. 6. Geneva: International Labur Organization.

53. MILL, John Stuart. (1976). On politics and society. Glasgow: Fontana Press. 412 pp.

54. MURO, Mark, MAXIM, Robert \& WHITON, Jacob. (2019). Automation and Artificial Intelligence: How Machines are Affecting People and Places. Washington: Metropolitan Policy Program. 108 pp.

55. NELSON, Richard \& PHELPS, Edmund. (1966). Investment in Humans, Technological Diffusion and Economic Growth. The American Economic Review, Vol. 56, № 1/2. Pp. 69-75. www.jstor.org/stable/1821269

56. OECD (2016). "The Risk of Automation for Jobs in OECD Countries, A Comparative Analysis". OECD Social, Employment and Migration Working Papers, №. 189. Paris: OECD Publishing. http://dx.doi.org/10.1787/5jlz9h56dvq7-en

57. OECD (2019). OECD Employment Outlook 2019: The Future of Work. Paris: OECD Publishing. https://doi.org/10.1787/9ee00155-en

58. PASINETTI, Luigi. (1981). Structural Change and Economic Growth. Cambridge: Cambridge University Press. 300 pp.

59. PÉREZ, Carlota. (2002). Technological revolutions and financial capital: the dynamics of bubbles and Golden ages. Cheltenham (UK): Edward Elgar. 119 pp.

60. PETERS, Bettina, HUD, Martin, DACHS, Bernhard \& KÖHLER, Christian. (2017). Employment Effects of Innovations over the Business Cycle: Firm-Level Evidence from European Countries, Beiträge zur Jahrestagung des Vereins für Socialpolitik. http:// dx.doi.org/10.2139/ssrn.2912140

61. PIANTA, Mario. (2000). The employment impact of product and process innovations (pp. 77-95). In: M. Pianta \& M. Vivarelli (Eds.), The employment impact of innovation: Evidence and Policy. Routledge. 
62. PIANTA, Mario. (2005). 'Innovation and Employment'. In: J. Fagerberg, D.C. Mowery and R.R. Nelson (Eds.), The Oxford Handbook of Innovation. Oxford: Oxford University Press. https://doi.org/10.4324/9780203458686

63. PIGOU, Arthur Cecil. (1933). The Theory of Unemployment. London: McMillan. 348 pp.

64. PORTER, Michael E. (1993). The competitive advantage of nations. Cambridge: Harvard Business School Management Programs. Pp. 73-93.

65. PREBISCH, Raúl \& MARTínEZ, Gustavo. (1949). El desarrollo económico de la América Latina y algunos de sus principales problemas. El trimestre económico, Vol. 16, № 63. Pp. 347-431. https://www.jstor.org/stable/20855070

66. PREBISCH, Raúl. (1950). The Economic Development of Latin America and its Principal Problems. New York: United Nations.

67. RICARDO, David. (1821). The Works and Correspondence of David Ricardo: Principles of Political Economy and Taxation. Cambridge: Cambridge University Press. 444 pp.

68. ROSENBERG, Nathan. (1976). Perspectives on technology. $1^{\text {a }}$ ed. Cambridge: Cambridge University Press. 364 pp.

69. ROSER, Max; ORTIZ-OSPINA, Esteban (2020) - "Income Inequality". Published online at OurWorldInData.org. Retrieved from: 'https://ourworldindata.org/income-inequality' [Online Resource]

70. SALTER, W.E.G. (1960). Productivity and Technical Change. $2^{\mathrm{a}}$ ed. Cambridge: Cambridge University Press. 198 pp.

71. SCHUMPETER, Joseph. (1942). Creative destruction. Capitalism, socialism and democracy. $3^{\text {a }}$ ed. New York: Harper \& Row. Pp. 82-85.

72. SCHUMPETER, Joseph. (1939). Business cycles. $1^{\mathrm{a}}$ ed. New York: McGraw-Hill. 466 pp.

73. SINGER, Hans. (1998). The terms of trade fifty years later-convergence and divergence. The South Letter, Vol. 30, № 1. Pp. 1-5.

74. TETHER, Bruce, MINA, Andrea, CONSOLI, Davide \& GAGLIARDI, Dimitri. (2005). A Literature review on skills and innovation. How does successful innovation impact on the demand for skills and how do skills drive innovation? A CRIC report for The Department of Trade and Industry, ESRC Centre for Research on Innovation and Competition: University of Manchester.

75. UNESCO (2005). UNESCO Science Report del 2005. Disponible en Internet: https:// unesdoc.unesco.org/ark:/48223/pf0000143007 Consultado: 30.08.2019.

76. VAN REENEN, John. (1997). Employment and technological innovation: Evidence from UK manufacturing firms. Journal of Labor Economics, Vol. 1, № 2. Pp. 255-284. www. jstor.org/stable/2535390

77. VIOLANTE, Giovanni L. (2008). Skill-biased technical change. The New Palgrave Dictionary of Economics. https://doi.org/10.1057/978-1-349-95121-5_2388-1

78. VIVARELLI, Marco. (1995). The Economics of Technology and Employment: Theory and Empirical Evidence. $1^{\text {a }}$ ed. Aldershot (UK): Edward Elgar.

79. VIVARELLI, Marco. (2007). Innovation and employment: a survey IZA Discussion Papers, No. 2621. Retrieved from: https://ssrn.com/abstract=969621 Consultado: 7.08.2019. 
80. VIVARELLI, Marco. (2012). Innovation, employment and skills in advanced and developing countries: A survey of the literature. Journal of Economic Issues, №. 6291. Pp. 1-61.

81. VIVARELLI, Marco. (2014). Innovation, employment and skills in advanced and developing countries: A survey of economic literature. Journal of Economic Issues, Vol. 48, No 1. Pp. 123-154. https://doi.org/10.2753/JEl0021-3624480106

82. WEBB, Michael. (2019). The Impact of Artificial Intelligence on the Labor Market. (November 6, 2019). $61 \mathrm{pp}$ Available at SSRN: https://ssrn.com/abstract=3482150 or http://dx.doi.org/10.2139/ssrn.3482150

83. WELCH, Finis. (1970). Education in Production. Journal of political economy, Vol. 78. Pp. 312-27.

84. WESTKÄMPER, Engelbert. (2014). Towards the Re-Industrialization of Europe: A Concept for Manufacturing for 2030. Berlin: Springer. 124 pp.

85. WICKSELL, Knut. (1961). Lectures on Political Economy. London: Routledge \& Kegan. $1906 \mathrm{pp}$.

Para citar este artículo:
HEIJS, Joost y ARENAS, Guillermo. (2020). Innovación y empleo. Paradojas sociales y económicas. Teuken Bidikay, Vol. 11, № 16. Medellín: Politécnico Colombiano. Pp.55-89. doi: 10.33571/teuken.v11n16a3 
El perfeccionamiento de técnicas para asegurar el suministro de agua para el consumo y los cultivos es uno de los más importantes logros tecnológicos de los pueblos indígenas mesoamericanos. La recolección y almacenamiento del agua se hacía a través de canales o zanjas a cielo abierto o mediante depósitos subterráneos cuya función era el aprovechamiento del agua de lluvia, principal fuente para las obras hidráulicas de uso comunitario. Los aztecas desarrollaron sistemas de riego con canales, acequias, terrazas y chinampas que tuvieron como base el agua de sus lagos, ríos, manantiales y arroyos; de esta manera, las comunidades fueron ampliando la frontera de las áreas de cultivo, como crecía la población del imperio, y con ella sus necesidades. No en vano, la ingeniería moderna aún se sorprende de la precisión del cálculo con el que nuestros ancestros indígenas lograron encauzar las corrientes de agua, dosificar su consumo y almacenarla para los períodos de sequía.

Laura Guzmán M.

Politécnico Colombiano. 